\title{
Dynamic Hedging Based on Fractional Order Stochastic Model with Memory Effect
}

\author{
Qing Li, ${ }^{1,2}$ Yanli Zhou, ${ }^{3}$ Xinquan Zhao, ${ }^{2}$ and Xiangyu Ge ${ }^{4}$ \\ ${ }^{1}$ Academy of Mathematics and Systems Science, Chinese Academy of Sciences, Beijing 100190, China \\ ${ }^{2}$ School of Statistics and Mathematics, Zhongnan University of Economics and Law, Wuhan 430073, China \\ ${ }^{3}$ School of Finance, Zhongnan University of Economics and Law, Wuhan 430073, China \\ ${ }^{4}$ Wuhan Technology and Business University, Wuhan 430065, China \\ Correspondence should be addressed to Yanli Zhou; ylzhou8507@gmail.com and Xiangyu Ge; xiangyu_ge@163.com
}

Received 4 April 2016; Revised 18 May 2016; Accepted 22 May 2016

Academic Editor: Josè A. Tenereiro Machado

Copyright (c) 2016 Qing Li et al. This is an open access article distributed under the Creative Commons Attribution License, which permits unrestricted use, distribution, and reproduction in any medium, provided the original work is properly cited.

\begin{abstract}
Many researchers have established various hedge models to get the optimal hedge ratio. However, most of the hedge models only discuss the discrete-time processes. In this paper, we construct the minimum variance model for the estimation of the optimal hedge ratio based on the stochastic differential equation. At the same time, also by considering memory effects, we establish the continuous-time hedge model with memory based on the fractional order stochastic differential equation driven by a fractional Brownian motion to estimate the optimal dynamic hedge ratio. In addition, we carry on the empirical analysis to examine the effectiveness of our proposed hedge models from both in-sample test and out-of-sample test.
\end{abstract}

\section{Introduction}

Hedge is one of the most important functions of futures; thus how to obtain the optimal hedge ratio becomes the key issue in hedge strategy. The earlier literatures mainly lay stress on estimating the hedge ratio by means of the discrete-time econometric models, such as the ordinary least squares model (OLS) [1-3], the minimum variance model $(\mathrm{MV})[4,5]$, the vector autocorrelation regressive model (VAR) [5], the error correction model (ECM) [6], and the vector error correction model (VECM) [7]. Recently, by taking account of the dynamic relationship between the spot and the futures returns, many researches begin to employ much complicated models to estimate the hedge ratio, for instance, the bivariate generalized autoregressive condition heteroscedasticity model (BGARCH) [8] and Markov regime switching model (MRS) $[9,10]$.

However, many of the complicated hedge models (such as the MV model based on VECM model, GARCH model, threshold GARCH model, and BGARCH model) cannot perform better than the simple OLS model or result in little improvement and even bad results [11]. Moreover, the existing complicated models may also cause the high cost of computation. Liu et al. [12] discussed the problem of the rollover hedge strategy for the long-term exposure of a well-diversified portfolio. The purpose of this paper is to propose a new method for estimating the hedge ratio under the condition that the price processes of the spot and the futures are continuous-time processes, and the corresponding continuous-time processes are denoted by the stochastic differential equations.

A lot of the financial variables with long memory effects have been found $[13,14]$. Garzarelli et al. proved the existence of memory effects in the stock price series by using the conditional probability approach and measured the extent of long memory [15]. Most frequently, the memory effect is measured by the autocorrelation function, and then Hurst index is introduced to measure the memory effect by Hurst [16]. Hurst index is often denoted by $H(0<H<1)$. In the case of $0<H<0.5$, time series has negative correlation and antipersistent behavior, which is called shortdependence memory; in the case of $H=0.5$, the time series have no dependence; in the case of $0.5<H<1$, time series has positive correlation and persistent behavior, which is 
the long-dependence memory. Persistent behavior was called "Joseph Effect" by Mandlebrot and Mandelbrot and Wallis [17]. Li and Duan [18] investigated the impact of correlated noises on dynamical systems by considering Fokker-Planck type equations under the fractional white noise measure. Cajueiro and Tabak [19] and Cajueiro and Tabak [20] also found the memory effects existing in the financial markets. $\mathrm{Li}$ et al. [21] constructed a fractional order stochastic differential equation model by adding the stochastic process into the fractional ordinary differential equation to price European option.

This paper attempts to derive the dynamic optimal hedge model based on the minimum variance (MV) hedge model. We describe the stock and futures price processes by using the mathematical instrument of stochastic differential equations. In addition, by considering the memory effects denoted by fractional Brownian motion, we continue to improve the hedge model by using the fractional order stochastic differential equations driven by fractional Brownian motion to estimate the optimal hedge ratio.

The rest of this paper is organized as follows. In Section 2, we give the hedge model of minimum variance (MV model), and according to the idea of the MV model, the optimal hedge model based on continuous-time process is then established, which is the stochastic differential equation model (SDE-MV model). In Section 3, the optimal hedge ratio is obtained under the continuous-time process with memory based on the fractional order stochastic differential equations driven by fractional Brownian motion (FSDE-MV model). In Section 4 , the empirical analysis is proposed to examine the hedge effectiveness of the SDE-MV and FSDE-MV models by using the financial data of the stock index futures and the stock index fund in China. In Section 5, several conclusions are given about our proposed hedge models.

\section{Dynamic Hedge Based on Continuous-Time Model}

2.1. Minimum Variance Hedge Model (MV Model). Lindahl [4] and Hsu et al. [22] give the conventional risk-minimizing hedge ratio under the condition that the joint distribution of spot and futures returns remains the same over time. Let $S_{t}$ and $F_{t}$ be the spot price and futures price at time $t$, respectively, and we can establish the portfolio $\pi$ as follows:

$$
\pi_{t}=S_{t}-h F_{t}
$$

where $h$ is the hedge ratio.

The corresponding differential form of the portfolio $\pi$ is

$$
d \pi=d(S-h F)=d S_{t}-h d F_{t} .
$$

We then can obtain the variance of the asset portfolio $\pi$ :

$$
\begin{aligned}
\operatorname{Var}(d \pi)= & \operatorname{Var}\left(d S_{t}-h d F_{t}\right) \\
= & \operatorname{Var}\left(d S_{t}\right)+h^{2} \operatorname{Var}\left(d F_{t}\right) \\
& -2 h \operatorname{Cov}\left(d S_{t}, d F_{t}\right) .
\end{aligned}
$$

In order to get the optimal hedge ratio, let the derivative of variance of the portfolio be equal to 0 ; namely,

$$
\frac{d \operatorname{Var}(d \pi)}{d h}=2 h \operatorname{Var}\left(d F_{t}\right)-2 \operatorname{Cov}\left(d S_{t}, d F_{t}\right)=0
$$

We solve (4) and get

$$
h^{*}=\frac{\operatorname{Cov}\left(d S_{t}, d F_{t}\right)}{\operatorname{Var}\left(d F_{t}\right)} .
$$

And it is easy to verify that the two-order derivative of variance is greater than 0 :

$$
\frac{d^{2} \operatorname{Var}(d \pi)}{d h^{2}}=2 \operatorname{Var}\left(d F_{t}\right)>0 .
$$

We thus obtain that $h$ is the minimum value, because of $\operatorname{Cov}(d S, d F)=\rho \sigma_{S} \sigma_{F}$, wherein $\rho$ is the correlation coefficient of the spot price and the futures price.

Therefore the optimal hedge ratio is

$$
h_{t}^{*}=\frac{\operatorname{Cov}\left(d S_{t}, d F_{t}\right)}{\operatorname{Var}\left(d F_{t}\right)}=\rho \frac{\sigma_{S}}{\sigma_{F}} .
$$

The hedge effectiveness can be denoted by the percentage reduction in variance for the hedged spot relative to the unhedged spot (see [2]) and usually measured by $e^{*}$ :

$$
e^{*}=\frac{\sigma_{S}^{2}-\sigma_{\pi}^{2}}{\sigma_{S}^{2}}=1-\frac{\sigma_{\pi}^{2}}{\sigma_{S}^{2}}
$$

which means that the larger the value of the effectiveness index $e^{*}$, the better the effect of the hedge.

The optimal hedge strategy above is established under the assumption that the joint distribution of the spot and futures returns remains the same during the time. However, with the arrival of new information, the joint distribution of the spot and futures may be dynamic. In this case, the optimal hedge ratio is not a constant, and therefore the static hedging strategy is not suitable for the multiperiod hedge risk of spot. With the consideration of the information set at the time $t-\tau$, we can obtain the following optimal dynamic hedge ratio by minimizing risk of the hedged portfolio $\pi=S_{t}-h_{t-\tau} F_{t}$, or $d \pi=d S_{t}-h_{t-\tau} d F_{t}$ :

$$
h_{t-\tau}^{*}=\frac{\operatorname{Cov}\left(d S_{t-\tau}, d F_{t-\tau}\right)}{\operatorname{Var}\left(d F_{t-\tau}\right)},
$$

where $\tau, \tau=0,1,2, \ldots, n$ is the lag time. The dynamic optimal hedge ratio depends on the variance and covariance of the lag assets price and the optimal lag time can be obtained by the empirical analysis.

2.2. Hedge Model Based on Stochastic Differential Equation (SDE-MV Model). We assume that the financial prices of spot and the futures follow the Geometric Brownian motion. The stochastic differential equation of the stock price is as follows:

$$
d S_{t}=\mu_{S} S_{t} d t+\sigma_{S} S_{t} d B_{S}(t),
$$


where $\mu_{S}$ is the drift coefficient of the stock price and $\sigma_{S}$ is the diffusion coefficient of the stock price.

Futures price is subject to the following stochastic differential equation:

$$
d F_{t}=\mu_{F} F_{t} d t+\sigma_{F} F_{t} d B_{F}(t),
$$

where $\mu_{F}$ is the drift coefficient of the futures price and $\sigma_{F}$ is the diffusion coefficient of the futures price.

In (10) and (11), $d B_{S}(t), d B_{F}(t)$ are Wiener processes, $d t$. $d t=0, d B_{S}(t) \cdot d t=d B_{F}(t) \cdot d t=0, d B_{S}(t) \cdot d B_{S}(t)=d t$, $d B_{F}(t) \cdot d B_{F}(t)=d t, d B_{S}(t) \cdot d B_{F}(t)=\rho d t$, and $\rho$ is the correlation coefficient of the spot and futures price.

Lemma 1. The price processes of the spot and futures follow stochastic equation (10) and (11), and thus the optimal hedge ratio is

$$
h_{t}^{*}=\frac{\operatorname{Cov}\left(d S_{t}, d F_{t}\right)}{\operatorname{Var}\left(d F_{t}\right)}=\rho \frac{\sigma_{S}}{\sigma_{F}} \frac{S_{t}}{F_{t}} .
$$

And the $h_{t}^{*}$ lag of $\tau$ period is

$$
h_{t-\tau}^{*}=\frac{\operatorname{Cov}\left(d S_{t-\tau}, d F_{t-\tau}\right)}{\operatorname{Var}\left(d F_{t-\tau}\right)}=\rho \frac{\sigma_{S}}{\sigma_{F}} \frac{S_{t-\tau}}{F_{t-\tau}} .
$$

Proof 1 (Minimum Variance Approach). According to (5), we have that the optimal hedge ratio is $h^{*}=\operatorname{Cov}\left(d S_{t}, d F_{t}\right) /$ $\operatorname{Var}\left(d F_{t}\right)$, where

$$
\begin{aligned}
\operatorname{Cov}\left(d S_{t}, d F_{t}\right) & =E\left[\left(d S_{t}-E\left[d S_{t}\right]\right)\left(d F_{t}-E\left[d F_{t}\right]\right)\right] \\
& =E\left[\sigma_{S} S_{t} d B_{S}(t) \sigma_{F} F_{t} d B_{F}(t)\right] \\
& =\sigma_{S} \sigma_{F} S_{t} F_{t} E\left[d B_{S}(t) d B_{F}(t)\right] \\
& =\sigma_{S} \sigma_{F} S_{t} F_{t} \rho d t=\rho \sigma_{S} \sigma_{F} S_{t} F_{t} d t \\
\operatorname{Var}\left(d F_{t}\right) & =\sigma_{F}^{2} F_{t}^{2} d t .
\end{aligned}
$$

By substituting $\operatorname{Cov}\left(d S_{t}, d F_{t}\right)=\rho \sigma_{S} \sigma_{F} S_{t} F_{t} d t$ into the optimal hedge ratio, we get the result

$$
h_{t}^{*}=\frac{\operatorname{Cov}\left(d S_{t}, d F_{t}\right)}{\operatorname{Var}\left(d F_{t}\right)}=\frac{\rho \sigma_{S} \sigma_{F} S_{t} F_{t} d t}{\sigma_{F}^{2} F_{t}^{2} d t}=\rho \frac{\sigma_{S}}{\sigma_{F}} \frac{S_{t}}{F_{t}} .
$$

Proof 2 (Minimize Volatility). According to (10) and (11), we have

$$
\begin{aligned}
d \pi= & d S_{t}-h_{t} d F_{t} \\
= & \mu_{S} S_{t} d t+\sigma_{S} S_{t} d B_{S}(t) \\
& -h_{t}\left[\mu_{F} F_{t} d t+\sigma_{F} F_{t} d B_{F}(t)\right] \\
= & \left(\mu_{S} S_{t}-h_{t} \mu_{F} F_{t}\right) d t+\sigma_{S} S_{t} d B_{S}(t) \\
& -h_{t} \sigma_{F} F_{t} d B_{F}(t) \\
= & \left(\mu_{S} S_{t}-h_{t} \mu_{F} F_{t}\right) d t \\
& +\sqrt{\sigma_{S}^{2} S_{t}^{2}+h_{t}^{2} \sigma_{F}^{2} F_{t}^{2}-2 h_{t} \rho \sigma_{S} \sigma_{F} S_{t} F_{t}} d B(t) .
\end{aligned}
$$

Now let

$$
g(h)=h_{t}^{2} \sigma_{F}^{2} F_{t}^{2}-2 h_{t} \rho \sigma_{S} \sigma_{F} S_{t} F_{t}+\sigma_{S}^{2} S_{t}^{2},
$$

and in order to minimize the uncertainty of the portfolio, $g\left(h_{t}\right)$ should be equal to zero; that is, $g\left(h_{t}\right)=0$.

However, the value of delta is

$$
\begin{aligned}
\Delta & =\left[\left(-2 \rho \sigma_{S} \sigma_{F} S_{t} F_{t}\right)^{2}-4 \sigma_{F}^{2} F_{t}^{2} \sigma_{S}^{2} S_{t}^{2}\right] \\
& =4\left(\rho^{2}-1\right) \sigma_{S}^{2} \sigma_{F}^{2} S_{t}^{2} F_{t}^{2}<0 .
\end{aligned}
$$

For $\rho^{2}<1$, the equation $g\left(h_{t}\right)=0$ has no solutions. Then the function $g\left(h_{t}\right)$ can be transformed as follows:

$$
\begin{aligned}
g\left(h_{t}\right) & =h_{t}^{2} \sigma_{F}^{2} F_{t}^{2}-2 h_{t} \rho \sigma_{S} \sigma_{F} S_{t} F_{t}+\sigma_{S}^{2} S_{t}^{2} \\
& =\sigma_{F}^{2} F_{t}^{2}\left(h_{t}-\frac{\rho \sigma_{S} S_{t}}{\sigma_{F} F_{t}}\right)^{2}+\left(1-\rho^{2}\right) \sigma_{S}^{2} S_{t}^{2} .
\end{aligned}
$$

When $h_{t}^{*}=\rho\left(\sigma_{S} / \sigma_{F}\right)\left(S_{t} / F_{t}\right)$, the function $g\left(h_{t}\right)$ has the minimum value, and the corresponding minimum value is as follows:

$$
g_{\min }\left(h_{t}\right)=\left(1-\rho^{2}\right) \sigma_{S}^{2} S_{t}^{2}
$$

Thus,

$$
\begin{aligned}
d \pi= & \left(\mu_{S} S_{t}-h_{t} \mu_{F} F_{t}\right) d t \\
& +\sqrt{\sigma_{S}^{2} S_{t}^{2}+h_{t}^{2} \sigma_{F}^{2} F_{t}^{2}-2 h_{t} \rho \sigma_{S} \sigma_{F} S_{t} F_{t}} d B(t) \\
= & \left(\mu_{S}-\rho \mu_{F} \frac{\sigma_{S}}{\sigma_{F}}\right) S_{t} d t+\sqrt{1-\rho^{2}} \sigma_{S} S_{t} d B(t) .
\end{aligned}
$$

Therefore, we can obtain $E[d \pi]=\left(\mu_{S}-\rho \mu_{F}\left(\sigma_{S} / \sigma_{F}\right)\right) S d t$, $\operatorname{Var}[d \pi]=\left(1-\rho^{2}\right) \sigma_{S}^{2} S^{2} d t$.

2.3. Forecast of Hedge Ratio. According to the finance theory, the relationship between futures and the underlying spot is

$$
F_{t}=S_{0} e^{r\left(t-t_{0}\right)} .
$$

The stock price is as follows, according to the Ito's Lemma:

$$
S_{t}=S_{0} e^{\left(r-\sigma_{S}^{2} / 2\right)\left(t-t_{0}\right)+\sigma_{S} B(t)}
$$

where $S_{0}$ is the initial vale, $r$ is the riskless interest rate, $t$ is the present time, $t_{0}$ is the start time, $t-t_{0}$ is the time interval, $\sigma_{S}$ is the total year volatility, and $\sigma_{S}=\sigma_{\text {day }} \sqrt{t-t_{0}}$ and $T=t-t_{0}$ is the total trading days in one year.

Thus the forecast of the hedge ratio is

$$
\begin{aligned}
h_{t}^{*} & =\rho \frac{\sigma_{S}}{\sigma_{F}} \frac{S_{t}}{F_{t}}=\rho \frac{\sigma_{S}}{\sigma_{F}} \frac{S_{0} e^{\left(r-\sigma_{S}^{2} / 2\right)\left(t-t_{0}\right)+\sigma_{S} B(t)}}{S_{0} e^{r\left(t-t_{0}\right)}} \\
& =\rho \frac{\sigma_{S}}{\sigma_{F}} e^{-\sigma_{S}^{2} / 2\left(t-t_{0}\right)+\sigma_{S} B(t)} .
\end{aligned}
$$

The hedge ratio can be given by Monte Carlo simulation, and then the expectation of hedge ratio is

$$
E\left[h_{t}^{*}\right]=E\left[\rho \frac{\sigma_{S}}{\sigma_{F}} e^{-\sigma_{S}^{2} / 2\left(t-t_{0}\right)+\sigma_{S} B(t)}\right]=\rho \frac{\sigma_{S}}{\sigma_{F}} e^{-\sigma_{S}^{2} / 2\left(t-t_{0}\right)} .
$$




\section{Dynamic Hedge Based on Continuous-Time Model with Memory}

If the asset price is martingale or semimartingale, the asset price process is independent, and thus the price process can be depicted by a Brownian motion. However, the asset price processes are usually dependent, which means the price of next period depends on that of the previous periods. This phenomenon is called the memory effect, and the memory effect can be driven by a fractional Brownian motion. In this section, we establish the hedging model by considering the memory effect of uncertainty and then get the optimal hedge ratio based on the stochastic differential equation driven by fractional Brownian motion (FSDE) and derive the forecast formula of the hedge ratio.

3.1. Fractional Order Stochastic Differential Equation Driven by Fractional Brownian Motion (FSDE). Fractional Brownian motion is neither a Markov process nor semimartingales, but it is still a Gaussian process. There are several kinds of definition according to the previous research papers. This section gives the definition of fractional Brownian motion defined by referring to Mandelbrot and Wallis [17], which we use to construct our model in this paper.

Definition 2. Fractional Brownian motion $B_{H}(t)$ can be denoted by the classical Brownian motion $B(t)$ as follows:

$$
\begin{gathered}
B_{H}(t)=C_{H}\left[\int_{-\infty}^{0}\left((t-s)^{H-1 / 2}-(-s)^{H-1 / 2}\right) d B(s)\right. \\
\left.\quad+\int_{0}^{t}(t-s)^{H-1 / 2} d B(s)\right],
\end{gathered}
$$

where $C_{H}=1 / \Gamma(1 / 2+H), \Gamma$ is the Gamma function $\Gamma(\alpha)=$ $\int_{0}^{\infty} x^{\alpha-1} \exp (-x) d x$, and $H$ is the Hurst parameter, $0<H<1$. When $H=1 / 2, C_{H}=1$, and $B_{H}(t)=B(t)$, it becomes the classic Brownian motion.

The increment of the fractional Brownian motion is

$$
\begin{aligned}
& \Delta B_{H}(t)=\Delta B_{H}(t+\Delta t)-B_{H}(t) \\
& \quad=C_{H}\left[\int_{t}^{t+\Delta t}(t+\Delta t-s)^{H-1 / 2} d B(s)\right. \\
& \left.\quad+\int_{-\infty}^{t}\left((t+\Delta t-s)^{H-1 / 2}-(t-s)^{H-1 / 2}\right) d B(s)\right]
\end{aligned}
$$

when $H=1 / 2, \Delta B_{H}(t)=\Delta B_{H}(t+\Delta t)-\Delta B_{H}(t)=$ $\int_{t}^{t+\Delta t} d B(s)=\Delta B(t)$.

Proposition 3. Assume $B_{H}(t)$ and $0<H<1$ is a Fractional Brownian motion; we can get that $B_{H}(t)$ is Gaussian process, its mean value is zero, the variance is $t^{2 H}, B_{H}(t) \sim N\left(0, t^{2 H}\right)$, and $H$ is the Hurst parameter:

(1) Mean value function: $E\left(B_{H}(t)-B_{H}(0)\right)=0$.

(2) Autocovariance function: $C_{H}(t, s)=E\left(B_{H}(t) B_{H}(s)\right)=$ $(1 / 2)\left[|t|^{2 H}+|s|^{2 H}-|t-s|^{2 H}\right]$.
(3) Autocorrelation function: $\gamma_{H}(\tau)=(1 / 2)\left[(\tau+1)^{2 H}-\right.$ $\left.2 \tau^{2 H}+(\tau-1)^{2 H}\right]$.

(4) Autovariance function: $\operatorname{Var}\left(B_{H}(t)\right)=E\left[B_{H}(t)^{2}\right]=$ $t^{2 H}, t=s$.

Proposition 4. The increment of the fractional Brownian motion $\Delta B_{H}(t)=B_{H}(t+\Delta t)-B_{H}(t)$ has the following properties:

(1) $E\left[\Delta B_{H}(t)\right]=0$.

(2) $\operatorname{Cov}\left(\Delta B_{H}(t), \Delta B_{H}(t+s)\right)=(1 / 2)(\Delta t)\left(|s+1|^{2 H}+\mid s-\right.$ $\left.\left.1\right|^{2 H}-2|s|^{2 H}\right)$.

(3) $\operatorname{Var}\left(\Delta B_{H}(t)\right)=(\Delta t)^{2 H}, t=s$ in (2).

(4) $\Delta B_{H}(t) \sim N\left(0, \Delta t^{2 H}\right)$, which follows the normal distribution.

Definition 5. Fractional order stochastic differential equation driven by a fractional Brownian motion (FSDE) is defined as follows:

$$
d X=\mu(X, t)+\sigma(X, t) d B_{H}(t),
$$

where $H$ is the Hurst index, namely, the memory parameter, $B_{H}(t)$ is fractional Brownian motion, $\mu(X, t)$ is the drift parameter of stochastic process, and $\sigma(X, t)$ is the diffusion coefficient.

3.2. Hedge Model Based on Fractional Order Stochastic Differential Equation with Memory (FSDE-MV Model). Assume that the price of the spot and the futures follows a stochastic differential equation driven by the fractional Brownian motion, the price equation of the stock is

$$
d S=\mu_{S} S d t+\sigma_{S} S d B_{H_{S}}(t)
$$

where $H_{S}$ is the memory parameter of the spot price, $d B_{H_{S}}(t)$ is the fractional Brownian motion, $\mu_{S}$ is the drift coefficient of the stock process, and $\sigma_{S}$ is the diffusion coefficient of the stock process.

Also, the price equation of the futures is

$$
d F=\mu_{F} F d t+\sigma_{F} F d B_{H_{F}}(t),
$$

where $H_{F}$ is the memory parameter of the futures price, $d B_{H_{F}}(t)$ is fractional Brownian motion, $\mu_{F}$ is the drift coefficient of the futures price, and $\sigma_{F}$ is the diffusion coefficient of the futures price.

In (29) and (30), $d B_{H_{S}}(t)$ and $d B_{H_{F}}(t)$ are Wiener processes, $d t \cdot d t=0, d B_{H_{S}}(t) \cdot d t=d B_{H_{F}}(t) \cdot d t=$ $0,\left[d B_{H_{S}}(t)\right]^{2}=(d t)^{2 H_{S}},\left[d B_{H_{F}}(t)\right]^{2}=(d t)^{2 H_{F}}, d B_{H_{S}}(t)$. $d B_{H_{F}}(t)=\rho(d t)^{H_{S}+H_{F}}$, and $\rho$ is the correlation coefficient of the spot price and futures price.

Lemma 6. If the price processes of the spot and the futures follow the fractional order stochastic equations (29) and (30), the optimal hedge ratio is

$$
h_{t}^{*}=\frac{\operatorname{Cov}(d S, d F)}{\operatorname{Var}(d F)}=\rho \frac{\sigma_{S}}{\sigma_{F}} \frac{S_{t}}{F_{t}}(d t)^{H_{S}-H_{F}} .
$$


And the lag $\tau$ period of $h_{t}^{*}$ is

$$
h_{t-\tau}^{*}=\frac{\operatorname{Cov}\left(d S_{t-\tau}, d F_{t-\tau}\right)}{\operatorname{Var}\left(d F_{t-\tau}\right)}=\rho \frac{\sigma_{S}}{\sigma_{F}} \frac{S_{t-\tau}}{F_{t-\tau}}(d t)^{H_{S}-H_{F}}
$$

Proof 1 (Minimum Variance Approach). According to (5), we have the optimal hedge ratio:

$$
h^{*}=\frac{\operatorname{Cov}\left(d S_{t}, d F_{t}\right)}{\operatorname{Var}\left(d F_{t}\right)},
$$

where

$$
\begin{aligned}
\operatorname{Cov}\left(d S_{t}, d F_{t}\right) & =E\left[\left(d S_{t}-E\left[d S_{t}\right]\right)\left(d F_{t}-E\left[d F_{t}\right]\right)\right] \\
& =E\left[\sigma_{S} S_{t} d B_{H_{S}}(t) \sigma_{F} F_{t} d B_{H_{F}}(t)\right] \\
& =\sigma_{S} \sigma_{F} S_{t} F_{t} E\left[d B_{H_{S}}(t) d B_{H_{F}}(t)\right] \\
& =\rho \sigma_{S} \sigma_{F} S_{t} F_{t}(d t)^{H_{S}+H_{F}} \\
\operatorname{Var}\left(d F_{t}\right) & =\sigma_{F}^{2} F_{t}^{2}(d t)^{2 H_{F}} .
\end{aligned}
$$

By substituting $\operatorname{Cov}(d S, d F)=\rho \sigma_{S} \sigma_{F} S F(d t)^{H_{S}+H_{F}}$ into the optimal hedge ratio, we get

$$
\begin{aligned}
h^{*} & =\frac{\operatorname{Cov}\left(d S_{t}, d F_{t}\right)}{\operatorname{Var}\left(d F_{t}\right)}=\frac{\rho \sigma_{S} \sigma_{F} S_{t} F_{t} d t}{\sigma_{F}^{2} F_{t}^{2} d t} \\
& =\rho \frac{\sigma_{S}}{\sigma_{F}} \frac{S_{t}}{F_{t}}(d t)^{H_{S}-H_{F}} .
\end{aligned}
$$

Proof 2 (Minimize Uncertainty). According to (29) and (30), we have

$$
\begin{aligned}
d \pi & =d S-h d F=\mu_{S} S d t+\sigma_{S} S d B_{H_{S}}(t) \\
& -h\left[\mu_{F} F d t+\sigma_{F} F d B_{H_{F}}(t)\right]=\left(\mu_{S} S-h \mu_{F} F\right) d t+\sigma_{S} S d B_{H_{S}}(t) \\
& -h \sigma_{F} F d B_{H_{F}}(t)=\left(\mu_{S} S-h \mu_{F} F\right) d t \\
& +\sqrt{\sigma_{S}^{2} S^{2}(d t)^{H_{S}-H_{F}}+h^{2} \sigma_{F}^{2} F^{2}(d t)^{H_{F}-H_{S}}-2 h \rho \sigma_{S} \sigma_{F} S F} d B_{H}(t),
\end{aligned}
$$

where $H=\left(H_{S}+H_{F}\right) / 2$.

Let

$$
\begin{aligned}
g(h)= & \sigma_{S}^{2} S^{2}(d t)^{H_{S}-H_{F}}+h^{2} \sigma_{F}^{2} F^{2}(d t)^{H_{F}-H_{S}} \\
& -2 h \rho \sigma_{S} \sigma_{F} S F
\end{aligned}
$$

in order to minimize the uncertainty of the portfolio, $g(h)$ should be equal to zero.

However, the value of delta is

$$
\begin{aligned}
\Delta & =\left[\left(-2 \rho \sigma_{S} \sigma_{F} S F\right)^{2}\right. \\
& \left.-4 \sigma_{F}^{2} F^{2}(d t)^{H_{F}-H_{S}} \sigma_{S}^{2} S^{2}(d t)^{H_{S}-H_{F}}\right]=4\left(\rho^{2}-1\right) \\
& \cdot \sigma_{S}^{2} \sigma_{F}^{2} S^{2} F^{2}<0 .
\end{aligned}
$$

So the equation $g(h)=0$ has no solutions:

$$
\begin{aligned}
g(h)= & h^{2} \sigma_{F}^{2} F^{2}(d t)^{H_{F}-H_{S}}-2 h \rho \sigma_{S} \sigma_{F} S F \\
& +\sigma_{S}^{2} S^{2}(d t)^{H_{S}-H_{F}} \\
= & \sigma_{F}^{2} F^{2}(d t)^{H_{F}-H_{S}}\left[h^{2}-\rho \frac{\sigma_{S} S}{\sigma_{F} F}(d t)^{H_{S}-H_{F}}\right]^{2} \\
& +\left(1-\rho^{2}\right) \sigma_{S}^{2} S^{2}(d t)^{H_{S}-H_{F}} .
\end{aligned}
$$

When $h=\rho\left(\sigma_{S} / \sigma_{F}\right)(S / F)(d t)^{H_{S}-H_{F}}$, the function $g(h)$ has the following minimum value:

$$
\begin{aligned}
g_{\min }(h)= & \left(1-\rho^{2}\right) \sigma_{S}^{2} F^{2}(d t)^{H_{S}-H_{F}}, \\
d \pi= & \left(\mu_{S} S-h \mu_{F} F\right) d t \\
& +\sqrt{\sigma_{S}^{2} S^{2}+h^{2} \sigma_{F}^{2} F^{2}-2 h \rho \sigma_{S} \sigma_{F} S F} d B_{H}(t) \\
= & {\left[\mu_{S}-\rho \mu_{F} \frac{\sigma_{S}}{\sigma_{F}}(d t)^{H_{S}-H_{F}}\right] S d t } \\
& +\sigma_{S} \sqrt{1-\rho^{2}}(d t)^{\left(H_{S}-H_{F}\right) / 2} S d B_{H}(t) .
\end{aligned}
$$

Therefore, we can obtain $E[d \pi]_{H}=\left[\mu_{S}-\rho \mu_{F}\left(\sigma_{S}\right)\right.$ $\left.\left.\sigma_{F}\right)(d t)^{H_{S}-H_{F}}\right] S d t, \operatorname{Var}[d \pi]_{H}=\left(1-\rho^{2}\right) \sigma_{S}^{2} S^{2}(d t)^{2 H_{S}}$.

3.3. Forecast of Hedge Ratio. FBM model is not semimartingale, so the arbitrage exists in the market, which means the no-arbitrage pricing theory does not work on the FBM model [23]. However, in our paper the arbitrage only exists in the market of futures and its underlying spot (i.e., we assume that prices of futures and underlying spot follow the FBM model). Equation (22) is obtained by using the no-arbitrage futures pricing theory. However, the nonarbitrage equation (22) depicts the arbitrage between futures market and its underlying spot market, which is not the nonarbitrage only used in the futures market or in the spot market. Therefore, the nonarbitrage equation (22) still can be used in (41).

According to (19), the relationship between the futures and the spot, and (20) and (21), the forecast formula of the hedge ratio under uncertainty with memory is

$$
\begin{aligned}
h_{t}^{*} & =\rho \frac{\sigma_{S}}{\sigma_{F}} \frac{S_{t}}{F_{t}}(d t)^{H_{S}-H_{F}} \\
& =\rho \frac{\sigma_{S}}{\sigma_{F}} \frac{S_{t_{0}} e^{r\left(t-t_{0}\right)-\left(\sigma_{S}^{2} / 2\right)\left(t-t_{0}\right)^{2 H_{S}}+\sigma_{S} B_{H_{S}}(t)}}{S_{t_{0}} e^{r\left(t-t_{0}\right)}}(d t)^{H_{S}-H_{F}} \\
& =\rho \frac{\sigma_{S}}{\sigma_{F}}(d t)^{H_{S}-H_{F}} e^{-\left(\sigma_{S}^{2} / 2\right)\left(t-t_{0}\right)^{2 H_{S}}+\sigma_{S} B_{H_{S}}(t)} .
\end{aligned}
$$

And the expectation of the hedge ratio is

$$
\begin{aligned}
E\left[h_{t}^{*}\right] & =E\left[\rho \frac{\sigma_{S}}{\sigma_{F}}(d t)^{H_{S}-H_{F}} e^{-\left(\sigma_{S}^{2} / 2\right)\left(t-t_{0}\right)^{2 H_{S}}+\sigma_{S} B_{H_{S}}(t)}\right] \\
& =\rho \frac{\sigma_{S}}{\sigma_{F}}(d t)^{H_{S}-H_{F}} e^{-\left(\sigma_{S}^{2} / 2\right)\left(t-t_{0}\right)^{2 H_{S}}} .
\end{aligned}
$$


TABLE 1: Statistics summary for HS300 index fund and futures.

\begin{tabular}{lcccccc}
\hline CSI300 index & Mean & Std. dev & Skewness & Kurtosis & Jarque-Bera & Hurst index \\
\hline Spot & -0.000283 & 0.006187 & -0.227774 & 4.529064 & 63.74512 & 0.6485 \\
Futures (IMIF) & -0.000293 & 0.006295 & -0.026827 & 4.769744 & 78.50241 & 0.6454 \\
\hline
\end{tabular}

TABLE 2: Comparison of the effectiveness results of in-sample hedging.

\begin{tabular}{|c|c|c|c|c|c|}
\hline Methods & $E\left[h^{*}\right]$ & $E[d \pi]$ & $\operatorname{Std}(d \pi)$ & $e^{*}$ & $\begin{array}{c}\text { Variance reduction } \\
\text { over conventional } \\
\text { MV method (\%) }\end{array}$ \\
\hline Unhedged & 0 & $-2.76 e-04$ & 0.006190 & 0 & \\
\hline MV & 0.9348 & $-1.27 e-05$ & 0.001910 & 0.9049 & \\
\hline \multicolumn{6}{|c|}{ Hedge Portfolio A: $d \pi=d S_{t}-h_{t}^{*} d F_{t}$} \\
\hline SDE-MV & 0.9315 & $-1.19 e-05$ & 0.001906 & 0.9053 & $0.42 \%$ \\
\hline FSDE-MV & 0.9159 & $-1.63 e-05$ & 0.001910 & 0.9049 & $0.00 \%$ \\
\hline \multicolumn{6}{|c|}{ Hedge Portfolio B: $d \pi=d S_{t}-h_{t-1}^{*} d F_{t}$ (the best effectiveness) } \\
\hline SDE-MV & 0.9315 & $-1.73 e-05$ & 0.001903 & 0.9056 & $0.94 \%$ \\
\hline FSDE-MV & 0.9159 & $-2.17 e-05$ & 0.001907 & 0.9050 & $0.52 \%$ \\
\hline \multicolumn{6}{|c|}{ Hedge Portfolio C: $d \pi=d S_{t}-h_{t-2}^{*} d F_{t}$} \\
\hline SDE-MV & 0.9315 & $-2.02 e-05$ & 0.001905 & 0.9032 & $0.52 \%$ \\
\hline FSDE-MV & 0.9159 & $-2.38 e-05$ & 0.001910 & 0.9027 & $0.00 \%$ \\
\hline
\end{tabular}

Note: the effectiveness of the hedging models is evaluated by using the contemporaneous, one-day-later, and two-day-later prices, respectively, of which the respective results are reported in the hedge portfolios $\mathrm{A}, \mathrm{B}$, and $\mathrm{C}$.

\section{Empirical Results}

This section will examine the hedge effectiveness of our proposed hedge models by using the data of stock index futures and stock index fund in China. To address the effectiveness of our SDE-MV model and FSDE-MV model, it is necessary to consider for the case that the futures are less correlated with the underlying spot.

4.1. Data and Hedge Asset. Now we take the daily close price of CSI300 Stock Index, CSI300 stock index futures (IMIF) for the following empirical analysis. All the data are obtained from China Financial Futures Exchange (CFFE), and the data sample is from 16 April 2010 to 28 September 2012 including 600 observations. In this empirical analysis, we use $T=t-t_{0}$, $\Delta t=T / N$. If $T$ denotes one year and $N$ denotes 252 trading days in one year in China financial market, thus $d t \approx \Delta t=$ $1 / 252$. The Hurst index of the spot and futures is obtained by the $R / S$ analysis approach. The summary statistics for the logarithm returns on the spot and futures for each asset are shown in Table 1.

As we can see from the results in Table 1, the correlation coefficient of CSI300 index fund and CSI300 index futures is 0.9511, so we can hedge CSI300 index fund by CSI300 index futures. And the Hurst parameters of the spot and futures are 0.6485 and 0.6454 , respectively; thus it indicates that the prices of the spot and futures have memory effect.

4.2. In-Sample Comparison of Different Hedge Models. This subsection evaluates the in-sample hedging performance of the previous different models. A hedge portfolio is composed of one unit spot and $h_{t}$ unit futures. For comparison, the hedge effectiveness index $e^{*}$ with different lag hedge ratios $h_{t-\tau}^{*}, \quad \tau=0,1,2(\tau=0$ represents contemporaneous time, $\tau=$ 1 is 1-day-later lag time, and $\tau=2$ is 2-day-later lag time), and then evaluate the variance reduction over the conventional MV method, the hedge ratio of MV models in (7), the hedge ratio of SDE model in (13), and the hedge ratio of FSDE model in (32). Table 2 summarizes the numerical results of the insample hedging performance for the different models with different lag time.

Hedge portfolios A, B, and C in Table 2 give the empirical results of the hedge effectiveness based on three different hedge ratios with the contemporaneous, 1-day-later, and 2-day-later data, respectively. The numerical results show that the 1-day-later hedge model performs better than the contemporaneous and 2-day-later models. Both SDE model and FSDE model outperform the conventional MV model, with the improvement over the MV model by $0.94 \%$ and $0.52 \%$, respectively.

4.3. Application in Fund Hedging (Case of Less Correlation). To test our models and address the effectiveness of our SDE model and FSDE model, it may be much interesting by consideration and application when the futures are less correlated with the underlying asset. Hence, we compare the performance of an alternative model for the fund exposure of holding the CSI300 stock index fund (Fund Code: 270010, GF Funds Company) with CSI300 stock index futures (Contact: IF1106). Our data sample is started from 18 October 2012 to 
TABLE 3: Statistics summary for HS300 index fund and futures.

\begin{tabular}{lcccccc}
\hline Asset & Mean & Std. dev & Skewness & Kurtosis & Jarque-Bera & Hurst index \\
\hline CSI300 index fund & -0.000262 & 0.002988 & -2.368096 & 16.20809 & 1312.568 & 0.6596 \\
CSI300 index futures & -0.000428 & 0.006758 & -0.833057 & 6.986605 & 124.4597 & 0.6743 \\
\hline
\end{tabular}

TABLE 4: Comparison of the effectiveness results of in-sample hedging.

\begin{tabular}{|c|c|c|c|c|c|}
\hline Methods & $E\left[h^{*}\right]$ & $E[d \pi]$ & $\operatorname{Std}(d \pi)$ & $e^{*}$ & $\begin{array}{c}\text { Variance reduction } \\
\text { over static MV } \\
\text { method }(\%)\end{array}$ \\
\hline Unhedged & 0 & $-2.62 e-04$ & 0.002988 & 0 & \\
\hline MV & 0.3524 & $-1.12 e-04$ & 0.001798 & 0.6397 & \\
\hline \multicolumn{6}{|c|}{ Hedge portfolio A: $d \pi=d S_{t}-h_{t}^{*} d F_{t}$} \\
\hline SDE-MV & 0.3409 & $-1.01 e-04$ & 0.001795 & 0.6391 & $0.33 \%$ \\
\hline FSDE-MV & 0.3695 & $-8.80 e-05$ & 0.00174 & 0.6395 & $0.44 \%$ \\
\hline \multicolumn{6}{|c|}{ Hedge portfolio B: $d \pi=d S_{t}-h_{t-1}^{*} d F_{t}$ (the best effectiveness) } \\
\hline SDE-MV & 0.3409 & $-1.25 e-04$ & 0.001798 & 0.6403 & $0.66 \%$ \\
\hline FSDE-MV & 0.3695 & $-1.12 e-04$ & 0.001796 & 0.6411 & $0.88 \%$ \\
\hline \multicolumn{6}{|c|}{ Hedge portfolio C: $d \pi=d S_{t}-h_{t-2}^{*} d F_{t}$} \\
\hline SDE-MV & 0.3409 & $-1.20 e-04$ & 0.001805 & 0.6363 & $0.44 \%$ \\
\hline FSDE-MV & 0.3695 & $-1.06 e-04$ & 0.001803 & 0.6371 & $0.66 \%$ \\
\hline
\end{tabular}

TABLE 5: Comparison of the effectiveness results of out-of-sample hedging.

\begin{tabular}{|c|c|c|c|c|c|}
\hline Model & $E\left[h^{*}\right]$ & $E[d \pi]$ & $\operatorname{Var}(d \pi)$ & $e^{*}$ & Variance reduction over static MV model (\%) \\
\hline \multicolumn{6}{|c|}{ Hedge portfolio B: $d \pi=d S_{t}-h_{t-1}^{*} d F_{t}$ (the best effectiveness) } \\
\hline Unhedged & 0 & $3.28 e-04$ & 0.006090 & 0 & \\
\hline MV & 0.9348 & $3.80 e-05$ & 0.001834 & 0.9093 & \\
\hline SDE-MV & 0.959789 & $3.99 e-05$ & 0.001819 & 0.9108 & $1.63 \%$ \\
\hline FSDE-MV & 0.930363 & $4.36 e-05$ & 0.001839 & 0.9088 & $-0.55 \%$ \\
\hline
\end{tabular}

10 June 2011. The summary statistics for the logarithm returns on the CSI300 stock index fund and futures for each asset are reported in Table 3.

The correlation coefficient of CSI300 index fund and CSI300 index futures is $\rho=0.7987$, so we can hedge CSI300 index fund by using CSI300 index futures.

In Tables 2 and 4, we get the numerical results regarding the hedge effectiveness of different hedge models. By taking account of the lag period, we can evaluate the effectiveness of the hedge models with contemporaneous, 1-day-later, and 2day-later prices, respectively, and then obtain the result that the hedge effectiveness of 1-day-later price is the best in all the in-sample tests.

4.4. Out-of-Sample Comparison of Different Hedge Models. When a hedge model performs very well on the in-sample data, it may not necessarily achieve good out-of-sample performance. It is therefore necessary to determine whether the proposed model still works well in terms of its outof-sample hedging. Now we forecast the value of hedge ratios by employing the Monte Carlo simulation according to the prediction formula of (25) and (41) and then test the performance of the prediction.
For convenience, the assets for the out-of-sample test we use are CSI300 index and CSI300 index futures, and the parameters of history data have been shown in Table 1, where the 600 observations are taken from 16 April 2010 to 28 September 2012. The sample data we use for testing the hedge effectiveness of out-of-sample hedging is from 8 October 2012 to 3 April 2013 (120 observations).

From the results of Table 4 , the Hurst parameter of the spot is larger than that of the futures, and thus we hedge the out-of-sample spot by using the 1-day-later hedge model. The numerical results of hedge effectiveness are shown in Table 5.

Table 5 reports the 1-day-later out-of-sample evaluation results of different hedging models based on the forecast hedge ratios. The hedging effectiveness of the SDE-MV model outperforms the MV model and the FSDE model; thus the SDE-MV model performs better both in sample and out of sample when the Hurst parameter of the spot is larger than the futures.

\section{Conclusions and Futures Work}

We propose a series of hedge models to estimate the optimal hedge ratios by considering the uncertainty and the memory effect of the asset price and then present the forecast 
hedging ratios of the models including the conventional MV model, the SDE-MV model, and the FSDE-MV model. By the empirical analysis of the proposed hedge models, we conclude that both SDE model and FSDE model outperform the conventional MV model and the hedge effectiveness of 1-day-later price is the best in all the in-sample tests. The SDE-MV model performs better both in sample and out of sample when the Hurst parameter of the spot is larger than the futures.

Since our paper assumes that the volatility of the spot and futures is constant over time, it will be interesting if the work continues to improve the model of this paper in terms of dynamic volatility by considering the stochastic volatility model or GARCH model.

\section{Competing Interests}

The authors declare that there is no conflict of interests regarding the publication of this paper.

\section{Acknowledgments}

This research work is supported by the Chinese National Social Science Foundation (10BJY104) and the China National Natural Science Foundation (11526193).

\section{References}

[1] L. L. Johnson, "The theory of hedging and speculation in commodity futures," The Review of Economic Studies, vol. 27, no. 3, pp. 139-151, 1960.

[2] L. H. Ederington, "The hedging performance of the new futures markets," The Journal of Finance, vol. 34, no. 1, pp. 157-170, 1979.

[3] S. Figlewski, "Hedging performance and basis risk in stock index futures," The Journal of Finance, vol. 39, no. 3, pp. 657669, 1984.

[4] M. Lindahl, "Minimum variance hedge ratios for stock index futures: duration and expiration effects," Journal of Futures Markets, vol. 12, no. 1, pp. 33-53, 1992.

[5] W. Yang and D. E. Allen, "Multivariate GARCH hedge ratios and hedging effectiveness in Australian futures markets," Accounting \& Finance, vol. 45, no. 2, pp. 301-321, 2005.

[6] A. Ghosh and R. Clayton, "Hedging with international stock index futures: an intertemporal error correction model," Journal of Financial Research, vol. 19, no. 4, pp. 477-491, 1996.

[7] D. Lien and X. Luo, "Estimating multiperiod hedge ratios in cointegrated markets," Journal of Futures Markets, vol. 13, no. 8, pp. 909-920, 1993.

[8] T. H. Park and L. N. Switzer, "Bivariate GARCH estimation of the optimal hedge ratios for stock index futures: a note," Journal of Futures Markets, vol. 15, no. 1, pp. 61-67, 1995.

[9] L. Sarno and G. Valente, "The cost of carry model and regime shifts in stock index futures markets: an empirical investigation," Journal of Futures Markets, vol. 20, no. 7, pp. 603-624, 2000.

[10] A. Alizadeh and N. Nomikos, "A markov regime switching approach for hedging stock indices," Journal of Futures Markets, vol. 24 , no. 7, pp. 649-674, 2004.

[11] D. H. Chen, L. Bin, and C. Y. Tseng, "Hedging effectiveness of applying constant and time-varying hedge ratios: evidence from
Taiwan stock index spot and futures," Journal of Risk \& Control, vol. 1, no. 1, pp. 31-49, 2014.

[12] Y. Liu, W.-G. Zhang, R. Chen, and J. Fu, "Hedging long-term exposures of a well-diversified portfolio with short-term stock index futures contracts," Mathematical Problems in Engineering, vol. 2014, Article ID 843240, 13 pages, 2014.

[13] E. Panas, "Long memory and chaotic models of prices on the London Metal Exchange," Resources Policy, vol. 27, no. 4, pp. 235-246, 2001.

[14] B. J. West and S. Picozzi, "Fractional Langevin model of memory in financial time series," Physical Review E-Statistical, Nonlinear, and Soft Matter Physics, vol. 65, no. 3, Article ID 037106, pp. 37-106, 2002.

[15] F. Garzarelli, M. Cristelli, G. Pompa, A. Zaccaria, and L. Pietronero, "Memory effects in stock price dynamics: evidences of technical trading," Scientific Reports, vol. 4, article 4487, 2014.

[16] H. E. Hurst, "Long-term storage capacity of reservoirs," Transactions of the American Society of Civil Engineers, vol. 116, pp. 770-808, 1951.

[17] B. B. Mandelbrot and J. R. Wallis, "Noah, joseph, and operational hydrology," Water Resources Research, vol. 4, no. 5, pp. 909-918, 1968.

[18] C. J. Li and J. Q. Duan, "Impact of correlated noises on additive dynamical systems," Mathematical Problems in Engineering, vol. 2014, Article ID 678976, 6 pages, 2014.

[19] D. O. Cajueiro and B. M. Tabak, “The Hurst exponent over time: testing the assertion that emerging markets are becoming more efficient," Physica A: Statistical Mechanics and Its Applications, vol. 336, no. 3-4, pp. 521-537, 2004.

[20] D. O. Cajueiro and B. M. Tabak, "Ranking efficiency for emerging markets," Chaos, Solitons and Fractals, vol. 22, no. 2, pp. 349352, 2004.

[21] Q. Li, Y. L. Zhou, X. Q. Zhao, and X. Y. Ge, "Fractional order stochastic differential equation with application in European option pricing," Discrete Dynamics in Nature and Society, vol. 2014, Article ID 621895, 12 pages, 2014.

[22] C.-C. Hsu, C.-P. Tseng, and Y.-H. Wang, "Dynamic hedging with futures: a copula-based GARCH model," Journal of Futures Markets, vol. 28, no. 11, pp. 1095-1116, 2008.

[23] L. C. Rogers, "Arbitrage with fractional Brownian motion," Mathematical Finance, vol. 7, no. 1, pp. 95-105, 1997. 


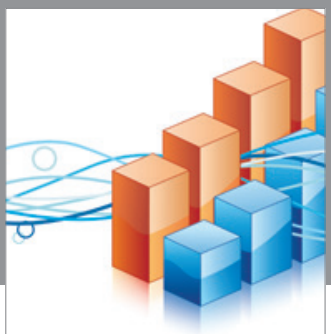

Advances in

Operations Research

vatem alat4

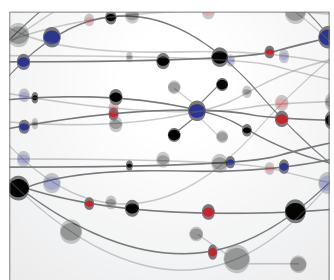

\section{The Scientific} World Journal
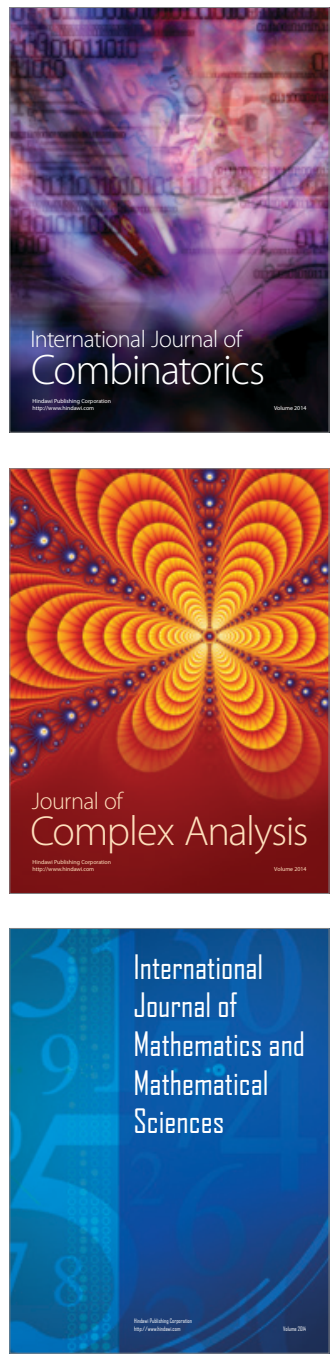
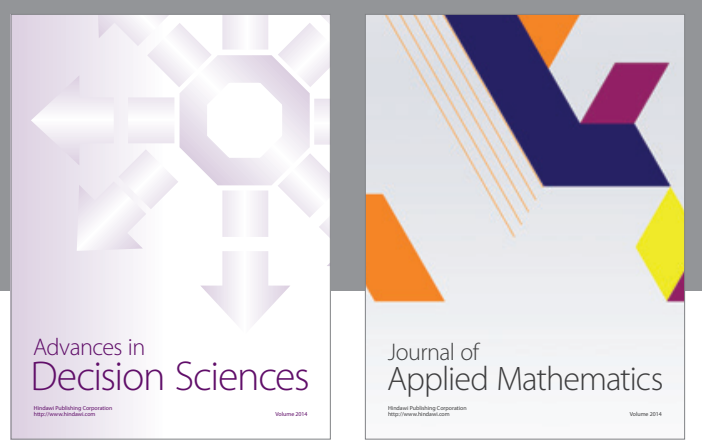

Algebra

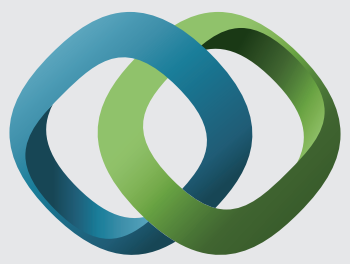

\section{Hindawi}

Submit your manuscripts at

http://www.hindawi.com
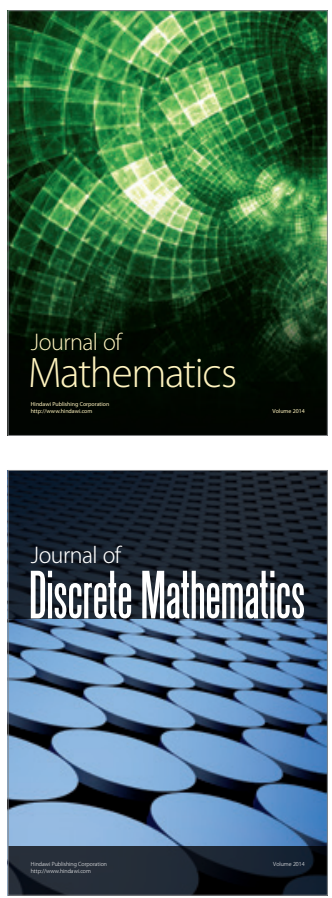

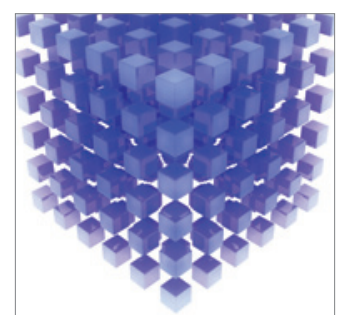

Mathematical Problems in Engineering
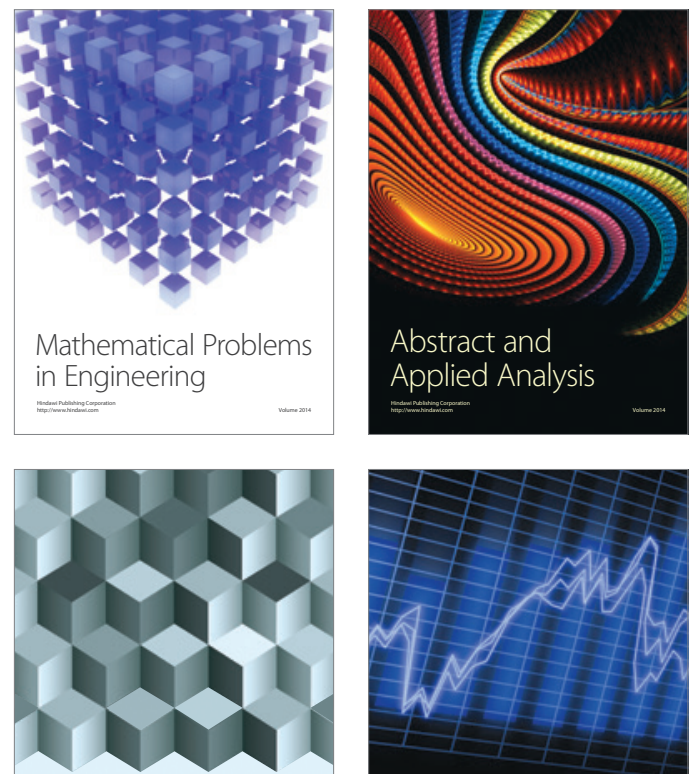

Journal of

Function Spaces

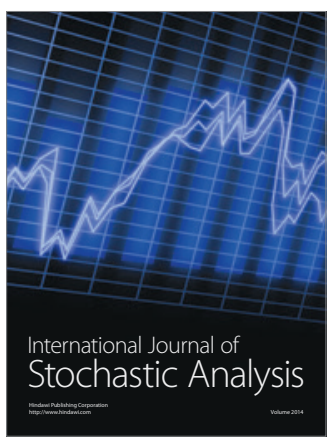

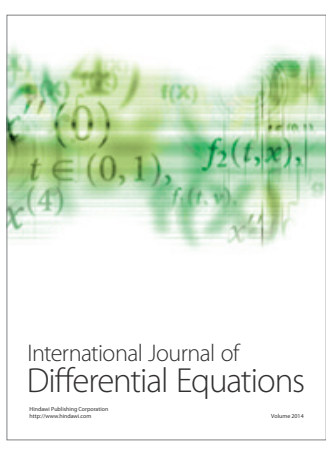
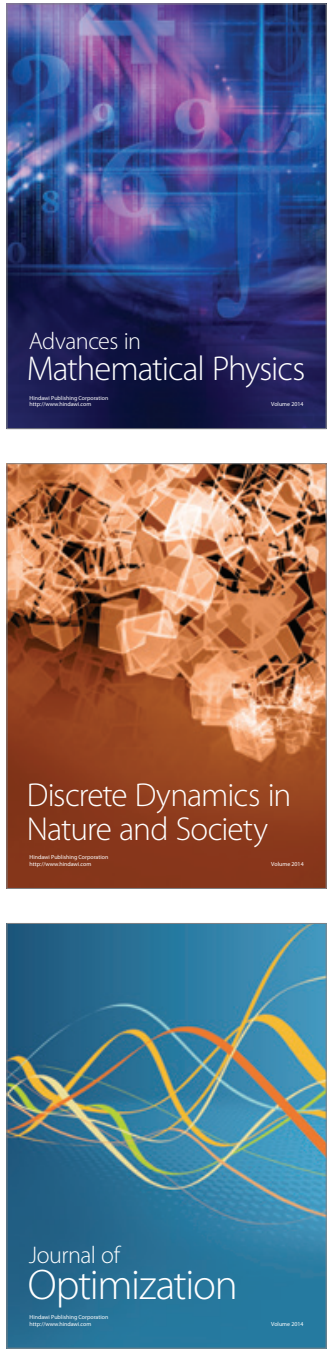